\title{
Energy Efficient Schemes for Wireless Sensor Network (WSN)
}

Sadia Batool,

* Mohtishim Siddique

\begin{abstract}
Conservation of energy is the main design issue in wireless sensor network (WSN) which is usually available at each node. Although different solutions have been introduced for typical wireless networks, cellular networks, MANET, and other short-range wireless local area networks, yet they are not often much feasible for a large scale WSN. For this purpose, multiple mobile sink nodes can be deployed to increase the life of sensor network. The purpose can be achieved by splitting the life time into equal time interval known as rounds. Similarly, by employing multiple sink nodes can also make the sensor network more energy efficient. Another way to make the sensor network energy efficient is to logically divide the deployment area into static clusters. By adopting the strategy of static cluster, energy consumption can be minimized. The two major wireless standards used by WSN are 802.15.4 and Zigbee [1] \& [2] . They are low-power protocols. Maximum distance is around 100m (at $2.4 \mathrm{GHz}$ ). However, performance is an issue. In order to assure the Wireless sensor network (WSN)s survivability and increase the lifetime of network in such environments, various energy efficiency schemes have been proposed in the literature. Energy is a valuable commodity in wireless networks due to the limited battery of the handy devices. The energy problem becomes stiffer in ad-hoc WSN).
\end{abstract}

Keywords: wireless sensor network (WSN)s (WSN), clustering scheme, energy efficient designs, and network lifetime.

\section{INTRODUCTION}

Typically WSN [ $\underline{3}]$ is composed of sink nodes and different numbers of wireless sensor nodes. All nodes have the ability to collect and process data independently. Wireless sensor systems made out of many sensor hubs which sense the physical environment regarding temperature, dampness, light, sound, vibration, and so forth. The fundamental assignment of sensor hub is to accumulate the information and data from the sensing field and send this to the end client by means of sink node. These sensor hubs can be conveyed on numerous applications. Now a day, wireless sensor network (WSN) is working to remove low power issues, communication, sensing, storage of energy, and computation.

Sadia Batool, *Mohtishim Siddique

Department of IT and CS

Minhaj University

Lahore, Pakistan

sadiamazhar19@gmail.com

mohtishimsiddique@hotmail.com 


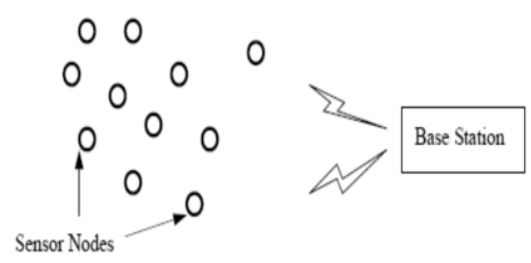

Figure 1 : Communication of Nodes and Sink Node

A Wireless sensor network (WSN) is a self-arranging system of nodes conveying among themselves utilizing radio signals, and sent in amount to sense, monitor and understand the physical world. Wireless Sensor hubs are called motes. WSN have a wide range of applications to different industries, field of science, transportation, infrastructure, and security. Usually, client node has limited source of energy and these are installed in a less responding areas. That is why, recharging is not possible frequently and for the performance of long-term applications, efficient energy solutions are required.

\subsection{Structure of wireless node}

A WSN is normally a fixed ad-hoc network [4] that comprises of hundreds of nodes. Each node has a transferring device (communication), a wireless transmitter/receiver (transducer) whose range is short, a low power processor and most important a limited source of energy (power). The transducer has an analog to digital converter(ADC)[]․ These nodes are capable of monitoring surrounding area and process the data collected and then sends that data towards sink node of that network. These sink nodes transmit the data received from nodes to some other control station. 


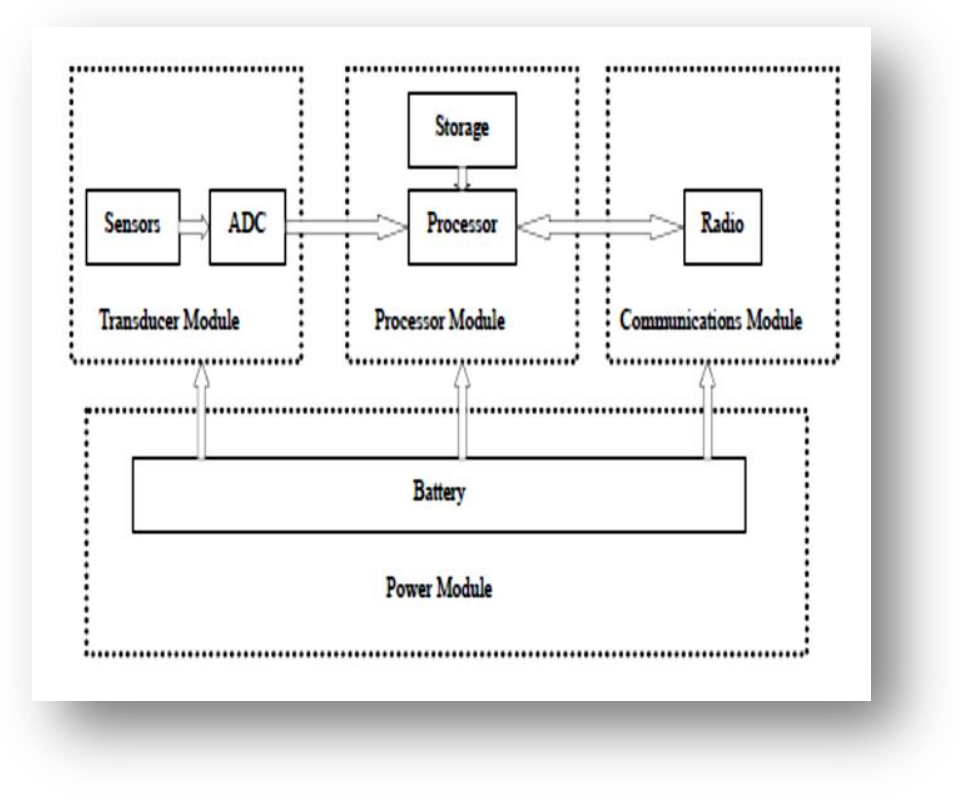

Figure 2: Structure of a Node

The function of these four modules (transducer, processor, communication, power) relies on the performance of node. A node is able to work in three dimensions: collection of data, head of cluster and data relay. If it works for the collection of data, then it will directly have passed the data to communication module to send. A cluster head or sink node has to collect and process data that is received from the members of network. Similarly, if a sensor is performing the task of a switch/relay then data will be received from neighbor nodes and will be sent again to sink or other nodes.

\subsection{Static Cluster}

Limited energy source is provided to Nodes that is why efficient energy utilization is very important in this area. In this paper, different schemes will be discussed to solve the problem of unbalanced energy utilization which results in less efficient in energy consumption. Concept of Static Cluster [ $\underline{6}$ ] is introduced which is done by dividing the areas into sub areas and then these sub areas are called static cluster. This division can control the coverage problem along with the efficient energy consumption.

\section{NOVEL LOAD BALANCING SCHEME}

Load balancing scheme is used in wireless sensor network if the aim is to provide the constant and reliable service. All those applications who generate periodic data for wireless sensor network requires the maximum possible life of a network. In wireless sensor networks(WSN), energy is assumed to be a strictly limited source. But the improved lifetime of the nodes is required to optimize the performance of energy consumption. That is why, Novel load balancing scheme [ $\underline{7}]$ is used to balance the consumption of energy in wireless sensor network (WSN)s.

\subsection{ENERGY PROBLEMS}


Every component of a system can be designed in an optimized way to reduce energy consumption. Energy is consumed when a node sense, communicate and process data. Efficient Algorithms can be designed to improve energy savings. Normally, the process which takes maximum power consumption is 'communication' rather than sensing or data processing. That is why distributed and localized algorithms are needed for different levels of communications. [5] . A simple model for the consumption of energy is proposed by [] in which energy dissipated by a node is simulated while sending and receiving data.

\subsection{DIFFERENT PROTOCOLS USED IN WSN}

Different protocols are being used in Wireless sensor network (WSN).

1. Protocol Stack

2. MAC protocol

3. TDMA based MAC Protocol

4. Power Aware Clustered TDMA(PACT)

5. Sensor-MAC

6. ALOHA with Preamble Sampling

In WSN, different solutions have been proposed for Medium Access Control protocol. Super Frame Time Scheduling[9] is designed to solve the issue of energy consumption. PACT (Power Aware Clustered TDMA) [10] is joint with an energy-efficient TDMA-based time schedule to reduce the overall utilization of energy in large-scale WSN.

One another advancement in Medium Access Control protocol was designed especially for WSN is S-MAC (Sensor-Medium Access Control). It is a mixture of reservation and contention based schemes planned to reduce the energy disorders that were produced due to the collisions occur in network, overheads of control packets, and idle listening[11].

One other protocol used in wireless networking is the Areal Locations of Hazardous Atmospheres(ALOHA) with Preamble Sampling scheme that is joined with the standard ALOHA protocol with the preamble sampling technique [12] minimize the error rate and maximize the performance.

A prime choice for the Wireless Sensor Networks is to use the Timed Division Multiple Access (TDMA) based MAC schemes (Medium Access Control) because radios can be turned off during their idle times in order to conserve energy by using E-TDMA.

The Energy-efficient TDMA (E-TDMA) is an extension to the classical TDMA to minimize the consumption of energy which is due to idle listening: it keeps the radio off when it has nothing to transmit.

\section{ROUTING PROTOCOLS}

1. Conventional [13]

a. Multi-hop Routing

b. Direct Communication with sink node

c. Static Clustering

2. Low Energy Adaptive Clustering Hierarchy -LEACH

3. Threshold Sensitive Energy Efficient Sensor Network[12]- TEEN

\section{TDMA AND ENERGY EFFICIENT TDMA}


In classical Timed Division Multiple Access, a node has to keep its radio on during the time slot allocated to it whether there is any data to send or not. That is why a lot of energy is consumed due to this idle work of sensing.

On the other hand, E-TDMA outperforms the basic TDMA to lessen the energy consumption due to idle state of the node. In this scheme, node keeps its radio off when it has no data to send during the time slot allocated to that node.

\subsection{BMA-MAC: AN ENERGY EFFICIENT SCHEME}

Keeping in mind the above mentioned problems, a new extension of MAC protocol is developed to reduce the consumption of energy. The core purpose is to reduce the energy wastage due to idle state of node and collisions occur during the communication while stabilizing a reasonable performance.

Rounds are introduced in BMA-MAC in which operation is divided it is done in LEACH [14]. Two phases are offered at each round which is Set-up phase and Steady-state phase.

\section{CONCLUSION}

In this paper, different schemes are discussed which are giving performance but the problem exists with the high consumption of energy. TDMA AND E-TDAM techniques are also discussed which are more energy efficient than other schemes. A new technique of BMA which is an extension to MAC has performed better than simple MAC protocol.

Basically it is planned for programs and applications which are event-driven, where if good events are detected only then data is transmitted to cluster head. If we analyze the performance of the Bit Map Assisted Medium Access Control, it is energy efficient than Time Division Multiple Access and Energy Efficient Time Division Multiple Access. Energy is saved from the idle periods and hence improved the overall performance.

Similarly, energy consumption of E-TDMA always improved than TDMA. E-TDMA scheme keep the node's radio off when there is no data to transmit. Similarly, both Bit Map Assisted Medium Access Control and Energy Efficient Time Division Medium Access can be joint to form a more efficient scheme in which E-TDMA can be used in large rounds while BMA can be utilized where rounds are small or medium. 


\section{REFERENCES}

[1]: Kumar, N., et al., GESTURE CONTOLLED ROBOTIC ARM USING WIRELESS

NETWORKS. GESTURE, 2016. 3(1).

[2]: Kinney, P. Zigbee technology: Wireless control that simply works. in Communications design conference. 2003.

[3]: Stallings, W., Wireless communications \& networks. 2009: Pearson Education India.

[4]: Gandham, S.R., et al. Energy efficient schemes for wireless sensor network (WSN)s with multiple mobile base stations. in Global telecommunications conference, 2003. GLOBECOM'03. IEEE. 2003. IEEE.

[5]: Li, J. and G.Y. Lazarou. A bit-map-assisted energy-efficient MAC scheme for wireless sensor network (WSN)s. in Proceedings of the 3rd international symposium on Information processing in sensor networks. 2004. ACM.

[6]: Ye, M., et al. EECS: an energy efficient clustering scheme in wireless sensor network (WSN)s. in PCCC 2005. 24th IEEE International Performance, Computing, and Communications Conference, 2005. 2005. IEEE.

[7]: Kim, H.-Y., An energy-efficient load balancing scheme to extend lifetime in wireless sensor network (WSN)s. Cluster Computing, 2016. 19(1): p. 279-283.

8.Heinzelman, W.R., A. Chandrakasan, and H. Balakrishnan. Energy-efficient communication protocol for wireless microsensor networks. in System sciences, 2000. Proceedings of the 33rd annual Hawaii international conference on. 2000. IEEE.

[9]: Sohrabi, K. and G.J. Pottie. Performance of a novel self-organization protocol for wireless ad-hoc sensor networks. in Vehicular Technology Conference, 1999. VTC 1999-Fall. IEEE VTS 50th. 1999. IEEE.

[10]: Pei, G. and C. Chien. Low power TDMA in large wireless sensor network (WSN)s. in Military Communications Conference, 2001. MILCOM 2001. Communications for NetworkCentric Operations: Creating the Information Force. IEEE. 2001. IEEE.

[11]: Iyengar, S.S. and R.R. Brooks, Distributed sensor networks: sensor networking and applications. 2016: CRC press.

[12]:Schurgers, C. and M.B. Srivastava. Energy efficient routing in wireless sensor network (WSN)s. in Military communications conference, 2001. MILCOM 2001. Communications for network-centric operations: Creating the information force. IEEE. 2001. IEEE.

[13]: Mahgoub, I. and M. Ilyas, Sensor network protocols. 2016: CRC press.

14.Razaque, A., et al. P-LEACH: Energy efficient routing protocol for Wireless sensor network $($ WSN)s. in 2016 IEEE Long Island Systems, Applications and Technology Conference (LISAT). 2016. IEEE. 